\title{
Nuclear Physics Research at ELI-NP
}

\author{
N.V. Zamfir ${ }^{*}$ \\ ELI-NP, "Horia Hulubei” National Institute for Physics and Nuclear Engineering (IFIN-HH), Bucharest-Magurele, Romania
}

\begin{abstract}
The new research facility Extreme Light Infrastructure - Nuclear Physics (ELI-NP) is under construction in Romania, on the Magurele Physics campus. Valued more than 300 Meuros the center will be operational in 2019. The research center will use a high brilliance Gamma Beam and a High-power Laser beam, with unprecedented characteristics worldwide, to investigate the interaction of very intense radiation with matter with specific focus on nuclear phenomena and their applications. The energetic particle beams and radiation produced by the $2 \times 10 \mathrm{PW}$ laser beam interacting with matter will be studied. The precisely tunable energy and excellent bandwidth of the gamma-ray beam will allow for new experimental approaches regarding nuclear astrophysics, nuclear resonance fluorescence, and applications. The experimental equipment is presented, together with the main directions of the research envisioned with special emphasizes on nuclear physics studies.
\end{abstract}

\section{Overview}

Extreme Light Infrastructure (ELI) Project was initiated in 2005, aiming to build the most powerful laser in history and to use it for generating applications unprecedented in science, as well as in industry and medicine. ELI became part of the 2006 Roadmap of ESFRI (36 European mega-projects of scientific research) [1], as a project aiming at the construction of a European and international research center at the highest level, focused on ultra high power lasers, laser-matter interaction and secondary sources of radiation. In 2009 the European Commission decided to implement this project in Czech Republic, Hungary and Romania, three complementary pillars, using Structural Funds.

In 2012 European Commission approved the Romanian application to host the nuclear pillar of the ELI project and the implementation started in January 2013. The new Center, Extreme Light Infrastructure - Nuclear Physics (ELI-NP) will be operational in 2019.

Selected by the most important science committees in Nuclear Physics in Europe - NuPECC - in the Nuclear Physics Long Range Plan in Europe [2] as a major facility, the ELI-NP infrastructure comprises two main components:

- A very High Power Laser System (HPLS), with two 10 PW laser arms based on Optical Parametric Chirped Pulse Amplification (OPCPA) able to reach intensities of $10^{23} \mathrm{~W} / \mathrm{cm}^{2}$ and electrical fields of $10^{15} \mathrm{~V} / \mathrm{m}$, constructed by an association between Thales Optronique France and Thales Romania;
- A very intense $\left(10^{13} \gamma / \mathrm{s}\right)$, brilliant Gamma Beam System (GBS), $\sim 0.1 \%$ bandwidth, with $\mathrm{E}_{\gamma}$ up to 19.5 $\mathrm{MeV}$, which is obtained by Compton back scattering of a laser light off a very brilliant, intense, classical electron beam $\left(\mathrm{E}_{\mathrm{e}}\right.$ up to $720 \mathrm{MeV}$ ) produced by a warm LINAC. GBS is constructed by EuroGammas Consortium composed of leading research institutions and private companies in accelerator and laser technologies: INFN Italy, University "La Sapienza" - Italy, CNRS - France, ALSYOM - France, ACP Systems S.A.S.U. - France, COMEB Srl -Italy, ScandiNova Systems - Sweden.

The combination of these equipment will create a new European laboratory with a broad range of science covering frontier fundamental physics, new nuclear physics and astrophysics, as well as applications in various fields [3].

\section{Current Status}

The ELI-NP project, valued at $311 \mathrm{M}$ Euros, is financed by Structural Funds and Romanian national budget.

The first phase of the project (2013-2016) was finalized with the construction of the facility, shown in Fig. 1, which covers approximately $33,000 \mathrm{~m}^{2}$ of high quality, energy efficient buildings, mostly dedicated to the highpower laser system, gamma-beam system and instrumentation for experiments, distributed in eight experimental areas:

- E1: laser induced nuclear reactions;

- E2: nuclear resonance fluorescence and applications;

- E3: positrons beams;

\footnotetext{
Corresponding author: victor.zamfir@eli-np.ro
} 
- E4 and E5: accelerated particle beams induced by HPLS (0.1 and 1 PW, respectively) at high repetition rates;

- E6: intense electron and gamma beams induced by high power laser beams;

- E7: experiments with combined laser and gamma beams;

- E8: nuclear reactions induced by high energy gamma beams.

Fig. 1. ELI-NP buildings.

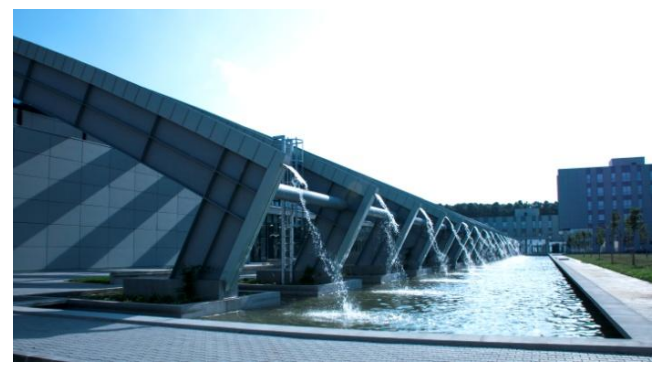

The civil construction comprises an office building, a guest house and a canteen.

Considering the unique features of the high-power laser and gamma beams, the scientific program was ellaborated : $E L I-N P$ White Book [4] is a result of a large international collaboration of more than 100 scientists from 30 countries. Following the general program, the Technical Design Reports (TDRs) were finalized and approved by ELI-NP International Scientific Advisory Board, in June 2015, and published in 2016 [5].

The second phase of the implementation of the project has received the approval of the European Comission on February $5^{\text {th }}, 2016$.

ELI-NP entered the second phase (2016-2019) with the installation and testing, in a progressive manner, of various components of the big equipment and experimental set-ups. The first laser beam, of a lower power, will be obtained by the end of 2017. The tests are going to last for two years, the infrastructure becoming fully operational in the summer of 2019, when all the parameters and full capacity of the large equipment will be reached. Figure 2 shows the laser hall with 2 arms of $10 \mathrm{PW}$ each installed.

Fig. 2. The ELI-NP Laser Room with the two arms of $10 \mathrm{PW}$ each.

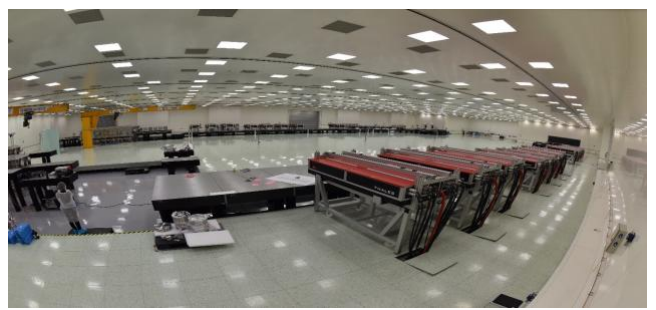

From the point of view of Human Resources, ELI-NP is also halfway along the road: in 2019, the research teams will consist in 324 employees, out of which 275 researchers. At this time (September 2017), our team consists of 149 employees, out of which 100 research and technical personnel from Romania and 20 other countries. Various positions are advertised all over the world in order to attract the best competencies, while the social impact of the project in Romania is also important to counteract the country's brain drain, by providing motivation for top young researchers to remain in the country.

By the end of the implementation phase (in 2019 and even earlier), there will be established a European Research Infrastructure Consortium - ELI-ERIC, a legal entity functioning in accordance with EU legal framework and benefiting from certain flexibility in regard with the rules of functioning. ELI-ERIC is meant to contribute significantly to strengthening and promoting leading European scientific research worldwide. Currently, the foundations of ELI-ERIC are being set-up through the preparation and submission of the documentation that will be the basis for issuing the European Commission Decision on establishing the Consortium (the Consortium Statutes and the TechnicalScientific Description of ELI infrastructure). The Romanian Government has approved, on 2 June 2016, the Memorandum on Romania's participation as a host Member State in the European Consortium for Research Infrastructure "Extreme Light Infrastructure" - ELIERIC.

\section{Scientific Program}

ELI-NP has the potential to be, for many years, in the forefront of worldwide science from theoretical physics to biology. The project has a great flexibility to cover various interdisciplinary areas, as a consequence of the possibility to employ, simultaneously in experiments, multiple radiation types, produced by equipment that will be unique at the moment of entering operation.

The most advanced research infrastructure in the world focusing on photonuclear physics studies and applications, ELI-NP will provide new opportunities for the study of fundamental processes that occur in lightmatter interaction [6] or in photonuclear reactions [7] and will actively promote its applications for the benefit of society, among which we can mention:

- Investigation of the high-power laser-matter interactions using nuclear physics methods in order to study the possibilities of obtaining electron, proton and heavy ion accelerated beams using lasers.

- The extremely high intensity of the laser beam will allow the study of fundamental physics phenomena anticipated by theory, such as vacuum birefringence and pair creation in intense electric fields.

- Investigation of nuclear structure via Nuclear Fluorecence Resonance (NRF) experiments. 
- Measurements of reactions cross sections of interest for astrophysics using photonuclear reactions $(\gamma, \mathrm{n}),(\gamma, \mathrm{p})$ or $(\gamma, \alpha)$

- New methods of identification and remote characterisation of nuclear materials will be investigated with application for homeland security (remote automatic scanning of transport conteiners) and nuclear material management.

- New ways of producing more efficiently radioisotopes currently used in medicine and the producing of newly proposed ones.

- Simultaneous use of the high intensity gamma and laser beams will enable fundamental physics studies as pair production in vacuum.

- Material studies using intense beam of moderated positrons produced by the $\left(\gamma, e^{+-}\right)$reaction.

- $\quad$ Other possible applications of the scientific results of the ELI-NP research activity could be: industrial tomography, active interrogation of materials and nuclear forensic with high-energy gamma beam and so on.

For the planned experimental set-ups a large variety of detector systems for $\gamma, \mathrm{n}, \mathrm{e}$, and $\mathrm{p}$ are planned. For example, for the experiments with gamma beam the following detectors are under construction:

- multi-detector array ELIADE (ELI-NP Array of DEtectors) will comprise 8 CLOVER Ge detectors plus additional 4 CLOVER type or $\mathrm{LaBr} 3(\mathrm{Ce})$ scintillator detectors mainly for the NRF studies [8];

- for experiments with gamma beam energy above neutron threshold - Gamma Above Neutron Threshold (GANT) [9], two different detector setups are proposed. The first is based on $3 \mathrm{He}$ tubes embedded in polyethylene for measurements of thermal neutrons (ELI-GANT-TN detector array), the second is an array of scintillators for measurements of gamma rays and fast neutrons (ELI-GANT-GN detector array) consisting of $\mathrm{LaBr} 3: \mathrm{Ce}$ and $\mathrm{CeBr} 3$ scintillation detectors for detection of gamma rays and BC501A liquid and GS20 6Li glass scintillators for detection of neutrons;

- High Efficiency Bragg Ionization Chamber (ELIBIC) with Si DSSD Detector System and Thick Gas Electron Multiplier (ELITHGEM) Detector System [10] for photofission studies;

- Large area Silicon Strip Detector (SSD) and a gas Time Projection Chamber detector read by an electronic readout system (e-TPC) [11].

Since the most advanced research topics are nowadays usually pursued in very large facilities, developed at international scale in order to serve a broader community of scientists, and given the unparalleled possibilities opened by this project, ELI-NP is establishing an Academic Forum open to universities and research institutions from Romania and abroad, having signed so far 65 Memorandums of Understanding for collaboration in implementing the project with prestigious institutions from all over the world.
ELI-NP is also aiming to become a catalyst for innovation in industry and the private sector, able to enhance transfer technology both to small and mediumsized enterprises and to large firms, and to train scientists and engineers in numerous disciplines associated with Extreme Light. In order to develop relations with the economic environment and high-tech industries, it was created Magurele High Tech Cluster (MHTC) which currently comprises more than 80 members, small and medium size companies, involved in the development of high technology products. ELI-NP maintains an ongoing dialogue with the business community, in order to identify the best levels of collaboration between researchers and producers.

This work is supported by the Project Extreme Light Infrastructure - Nuclear Physics (ELI-NP) - Phase I, a project co-financed by the Romanian Government and European Union through the European Regional Development Fund.

\section{References}

1. European Roadmap for Reserach Infrastructures Report 2006

https://ec.europa.eu/research/infrastructures/pdf/esfri lesfri_roadmap/roadmap_2006/esfri_roadmap_2006 en.pdf

2. NuPECC Long Range Plan 2010, Nuclear Physics: Perspectives for research in Europe, Science and Applications

http://www.nupecc.org/lrp2010/Documents//rp2010 _booklet_final.pdf

3. N.V. Zamfir, EPJ Web of Conferences 66, 11043 (2014)

4. http://www.eli-np.ro/documents/ELI-NPWhiteBook.pdf

5. Romanian Reports in Physics vol. 68 Supplement I,II (2016) (online: www.rrp.infim.ro)

6. N.V. Zamfir, Eur. Phys. J. Special Topics 223, 1221-1227 (2014)

7. D. Filipescu et al., Eur. Phys. J. A 51, 85(2015)

8. C.A. Ur et al., Romanian Reports in Physics, Vol. 68, Supplement, P. S483-S538 (2016)

9. F. Camera et al., Romanian Reports in Physics, Vol. 68, Supplement, P. S539-S619 (2016)

10. D.L. Balabanski et al., Romanian Reports in Physics, Vol. 68, Supplement, P. S621-S698 (2016)

11. O. Tesileanu et al., Romanian Reports in Physics, Vol. 68, Supplement, P. S699-S734 (2016) 